\title{
A DESCRIPTIVE EPIDEMIOLOGICAL STUDY ON MIGRAINE
}

\author{
POOJITHA MAMINDLA ${ }^{1}$, SHARANYA MOGILICHERLA ${ }^{1}$, DEEPTHI ENUMULA ${ }^{*}$, OM PRAKASH PRASAD ${ }^{2}$
}

${ }^{1}$ Department of Pharmacy Practice, Balaji Institute of Pharmaceutical Sciences, Warangal, Telangana, India. ${ }^{2}$ Department of Sri Sri Neuro Centre, Hanamkonda, Warangal, Telangana, India. Email: deepthi.e9@gmail.com/deepthi.enumula@learner.manipal.edu

Received: 01 May 2020, Revised and Accepted: 11 June 2020

ABSTRACT

Objective: The main objective was to carry out the observational study in migraine patients.

Methods: A prospective observational study was conducted for 6 months in 415 migraine patients with the prior approval from the Institutional Ethical committee. A predesigned pro forma was used to collect data such as socio-demographics of the patients, different types of migraine, triggering factors, and prescription pattern.

Results: Among 415 patients, the female patients ( $n=356,86 \%)$ outnumbered male patients ( $n=95,14 \%)$. The mean age of the study population was found to be $59 \pm 54$ years. In our study, we found that migraine without aura $(79 \%)$ was most common, followed by probable migraine without aura $(13.3 \%)$, chronic migraines (5\%), and migraine with aura (0.3\%). Grading is done according to International Headache Society into 0 , mild, moderate, and severe.

Conclusion: Compared to migraine with aura, migraine without aura is mostly seen in females than males due to fluctuating hormone levels, rural area is most effected due to exposure to triggers. Management includes pharmacological and non-pharmacological. Pharmacological therapy included nonsteroidal anti-inflammatory drugs agents, Triptans, and prophylactic therapy, and adjunctive therapy also prescribed.

Keywords: Migraine, Confirmatory analysis, Triggers, Prophylactic therapy, Adjunctive therapy.

(C) 2020 The Authors. Published by Innovare Academic Sciences Pvt Ltd. This is an open access article under the CC BY license (http://creativecommons. org/licenses/by/4. 0/) DOI: http://dx.doi.org/10.22159/ajpcr.2020.v13i8.38102

\section{INTRODUCTION}

Headache disorder is characterized by recurrent headaches, and it is the common nervous system disorder. Among all these, migraine headache is ubiquitous, disabling, and essentially treatable, but still underestimation and undertreated [1]. The name "migraine" originally comes from the Greek word "hemicranias," meaning "half of the head," is a neurovascular disease, representing one of the most striking features of the condition: That in many cases pain only affects one half of the head, characterized by a broad spectrum of symptoms $[2,3]$. Migraine attack can be triggered by a number of intrinsic or extrinsic factors such as environmental, dietary, or physiologic factors that can stimulate migraine activity [4]. In various regions, the socio-cultural factors play a major role in influencing the significance of triggering factors. Studies related to migraine triggers and its management are limited in India due to a lack of information [5]. The World Health Organization considered migraine as the major paroxysmal neurovascular disorder. The exact cause of a migraine attack is unknown, but it certainly lies within the central nervous system [6]. Migraine is a disorder which is characterized by multiple phases contains premonitory phase, aura phase, headache phase, postdrome phase, and interictal phase [7]. The association between subtypes of migraine was described by the International Headache Society (IHS) criteria [8]. Some prior studies have described that migraine may cause with some other illnesses and these occurrences is called as comorbidities to treat migraine, the comorbidities of migraine are important, because it can help in improving the treatment strategies and also understanding of the possible pathophysiology of migraine $[3,9]$. Cooccurrence of migraine subtypes and the migraine and tension-type headache within the same individual due to the lack of pathognomonic markers, the validity of the inclusion criteria and boundaries between migraine and also other subtypes of headache, classification of migraine has been delayed. The IHS criteria and Headache Classification Committee defined the association between the subtypes of migraine and its nature [8]. The pharmacological treatment of migraine patients with frequent severe headaches often requires both acute (abortive) and preventive treatment [10]. The main objective of our study was to carry out an observational study in migraine patients.

\section{METHODS}

\section{Study design and settings}

A prospective observational study was conducted for a period 6 months from March 2018 to August 2018 in Warangal area. An informed consent was taken from patients and patient representatives with the prior approval from Institutional Ethical Committee (BIPS/IEC/2018/P9)

\section{Study size}

A total of 415 Migraine patients were recruited into the study.

\section{Study criteria}

\section{Inclusion Criteria}

The following criteria were included in the study:

- All genders

- Age groups: $\geq 6-80$ years

- Patients with all sub-types of migraine headache.

Patients with comorbidities such as hypertension, stroke, coronary heart disease, subclinical vascular brain lesions, diabetes mellitus, lumber and cervical pain, asthma, epilepsy, fibromyalgia, and some psychiatric diseases such as depression, anxiety, schizophrenia, panic disorder, suicidal ideations, and bipolar disorder were included in the study.

\section{Exclusion criteria}

The following criteria were excluded from the study:

- Age group of below 5 years

- Patient with missing data. 
Study material

A predesigned pro forma was used to collect data on socio-demographics, personnel history, disease/headache status, phases of migraine, triggers of migraine, associated medical condition/comorbidity, medical and medication history, investigations, and prescription pattern in migraine patients. Data were collected and entered into MS Excel database, and statistical analysis was performed. The continuous data were represented in terms of frequency and percentages.

\section{RESULTS}

Socio-demographic characteristics of patients

Among 415 patients, the female patients $(n=356)$ outnumbered the male patients $(n=95)$. The mean age of the study population was found to be $59 \pm 54$ years, $76 \%$ patients were from rural area, $24 \%$ patients were from urban area, $2 \%$ of patients had a family history of migraine, and $50 \%$ of patients were normal weight, and the detailed information on socio-demographics is represented in Table 1.

\section{Types of migraine}

Of 415 patients, the highest number of patients $n=294$ was found to have migraines without aura type and the lowest number of patients $n=1$ had reported menstrual migraine type. The other details on different types of migraine are represented in Table 2.

Triggering factors, grading, and different phases in migraine patients

There are many triggering factors in single or combinational effecting the onset of migraine. In our study, we found that stress is the most triggering factor, followed by journey and change of weather. Grading

\section{Table 1: Socio-demographic characteristics of patients}

\begin{tabular}{|c|c|c|c|}
\hline \multicolumn{2}{|c|}{ Socio-demographic parameters } & Frequency (n) & Percentage \\
\hline \multicolumn{4}{|c|}{ Gender } \\
\hline \multicolumn{2}{|l|}{ Female } & 356 & 86 \\
\hline \multicolumn{2}{|l|}{ Male } & 95 & 14 \\
\hline \multicolumn{4}{|l|}{ Area } \\
\hline \multicolumn{2}{|l|}{ Urban } & 99 & 24 \\
\hline \multicolumn{2}{|l|}{ Rural } & 316 & 76 \\
\hline \multicolumn{4}{|l|}{ Family history } \\
\hline \multicolumn{2}{|l|}{ Positive } & 10 & 2 \\
\hline \multicolumn{2}{|l|}{ Negative } & 405 & 98 \\
\hline \multicolumn{4}{|l|}{ Age distribution } \\
\hline \multicolumn{2}{|l|}{$6-15$} & 13 & 3 \\
\hline \multicolumn{2}{|l|}{$16-25$} & 68 & 16 \\
\hline \multicolumn{2}{|l|}{$26-35$} & 130 & 31 \\
\hline \multicolumn{2}{|l|}{$36-45$} & 135 & 33 \\
\hline \multicolumn{2}{|l|}{$46-55$} & 42 & 10 \\
\hline \multicolumn{2}{|l|}{$56-65$} & 19 & 5 \\
\hline \multicolumn{2}{|l|}{$66-75$} & 8 & 2 \\
\hline \multicolumn{4}{|l|}{ BMI status } \\
\hline BMI status & BMI range & 65 & 16 \\
\hline Under weight & $<18.5$ & & \\
\hline Normal weight & $18.5-24.9$ & 209 & 50 \\
\hline Overweight & $25-29.9$ & 90 & 22 \\
\hline Obese & 30 and above & 51 & 12 \\
\hline
\end{tabular}

Table 2: Different types of migraine

\begin{tabular}{lll}
\hline Types of primary headache & Frequency (n=415) & Percentage \\
\hline Migraine & & \\
Migraine & 38 & 9 \\
Migraine without aura & 294 & 71 \\
Migraine with aura & 2 & 0.3 \\
Chronic migraine & 3 & 1 \\
Chronic migraine without aura & 17 & 4 \\
Probable migraine & 2 & 0.3 \\
Probable migraine without aura & 56 & 13 \\
Vestibular migraine & 2 & 0.3 \\
Menstrual migraine & 1 & 0.1 \\
\hline
\end{tabular}

done according to the IHS into 0, mild, moderate, and severe, we found that 141 patients were in severe, followed by 84 in moderate grade. There are one or more phases of migraine experienced by each patient; in our study, we have observed that the phase of associated features with headache was experienced by the majority of the patients. The details of triggering factors, grading, and phases of migraine are represented in Table 3 .

\section{Migraine with comorbidities}

Many comorbidities along with migraine were reported hypertension $(n=49)$, thyroid disorder $(n=39)$, cervical PIVD $(n=32)$, migraine related vertigo $(n=28)$, lumbar PIVD $(n=17)$, diabetes mellitus $(n=14)$, seizures $(n=10)$, ulcer $(n=7)$, epilepsy $(n=5)$, axonal neuropathy $(n=5)$, coronary artery disease $(n=5)$, benign paroxysmal positional vertigo $(n=3)$, psychiatric illness $(n=2)$, sinusitis $(n=2)$, cortical vein thrombosis $(n=2)$, asthma $(n=2)$, cervical spondylosis $(n=1)$, hemiparesthesia $(n=1)$, anemia $(n=1)$, Meniere's disease $(n=1)$, anxiety $(n=1)$, mouth deviation $(n=1)$, hearing impairment $(n=1)$, hematoma $(n=1)$, and tertiary ischemic stroke $(n=1)$.

\section{Prescription pattern in migraine patients}

There are the different class of drugs used for treating migraine using single/combination of two or three drugs which includes headache therapy drugs, prophylactic therapy, and adjunctive therapy. Nonsteroidal anti-inflammatory drugs (NSAIDs), triptans, and calcium channel blockers were the most predominantly prescribed drugs in our study. The prescription pattern of drugs used for treating migraine and its comorbidities are represented in Table 4.

\section{DISCUSSION}

Migraine headaches are primary headache disorders, which are called as stand-alone illnesses caused directly by the over activity and result from changes in chemical activity in the brain. Giles Elrington in Migraine: Diagnosis and management, stated migraine in two types with aura and without aura [11]. At the same time, we found subjects with probable, chronic, vestibular, and menstrual migraine with aura and without aura symptoms. In our study, we have observed that

Table 3: Triggering factors, grading, and different phases in migraine patients

\begin{tabular}{lll}
\hline Different parameters & Frequency (n) & Percentage \\
\hline Triggers & & \\
Stress & 312 & 22 \\
Change of weather & 237 & 17 \\
Sun light & 6 & 0.5 \\
Journey & 243 & 17 \\
Strong smells & 121 & 8.5 \\
Head bath & 131 & 9 \\
Sleep & 206 & 15 \\
Food & 114 & 8 \\
Menstruation & 25 & 2 \\
Grade & & \\
0 & 0 & $0 \%$ \\
1 & 0 & $0 \%$ \\
2 & 6 & $1 \%$ \\
3 & 6 & $1 \%$ \\
4 & 41 & $10 \%$ \\
5 & 84 & $20 \%$ \\
6 & 20 & $5 \%$ \\
7 & 12 & $3 \%$ \\
8 & 80 & $19 \%$ \\
9 & 141 & $34 \%$ \\
10 & 25 & $6 \%$ \\
Phases of migraine* & & $30 \%$ \\
Prodrome phase & 224 & $52 \%$ \\
Associated features with headache & 383 & $18 \%$ \\
Postdrome phase & 131 & \\
\hline
\end{tabular}

*Total number may vary because each patient may experience more than one phase 
Table 4: Prescription pattern of drugs in migraine patients

\begin{tabular}{lll}
\hline Type of therapy & Drugs & Frequency (n) \\
\hline Headache therapy & NSAIDs(+Domperidone) & 385 \\
& Triptans & 318 \\
Prophylactic & Antidepressants & 63 \\
Therapy & Antipsychotics & 10 \\
& Anticonvulsant & 204 \\
& Beta-blockers & 280 \\
Adjunctive & Ca channel blockers & 294 \\
therapy & Analgesics & 111 \\
Other therapy & Antacids & 380 \\
& Anti-emetics & 371 \\
& Vitamins & 117 \\
& Antibiotics & 1 \\
& Antiplatelets & 5 \\
& Antihistamine & 44 \\
& Antipyretic & 16 \\
& Anticoagulants & 1 \\
& Corticosteroids & 15 \\
& Antidiabetics & 3 \\
& Statins & 1 \\
& Anti-arrhythmic & 2 \\
& Angiotensin receptor blockers & 13 \\
& Diuretics & 7 \\
& Thyroid drugs & 2 \\
& PEG & 1 \\
& Cognitive enhancers & \\
& & \\
& & \\
& &
\end{tabular}

females are more effected with migraine than males with the age group between 25 and 35, because of fluctuating hormone levels such as fall in levels of estrogen and progesterone. Approximately two-thirds of women who experience migraines said their symptoms improve, and some worsen as they reach menopause, as some use hormone replacement therapies. Urban area subjects are affected less than the rural area in our study because of their occupation and lifestyle changes such as skipping meals, disturbed sleep patterns, intense lights, sounds or smells, and severe weather changes and stress. In general studies, hereditary is the main causative factor of migraine, but in our study, we have observed that only $2 \%$ are with a positive family history with headache as mentioned in the above because most headaches are due to their lifestyle factors, and environmental factors. The main reason for overweight in our study we observed is the chronic use of migraine medication but there is no link between migraine and obesity. Bigal et al. stated that headache is not associated with BMI but was associated with the frequency of headache attacks [12]. Stress is the main triggering factor in our study because most of the patients were job holders and complies with the study done by Yadav et al. [5]. Grading/severity of migraine is done by IHS grading scale, which contains four steps, including the "zero," "mild," "moderate," and "severe," and grading includes 0-10 grades. Based on the questionnaire, we gave grades for headache intensity. We found patients with different grades and majorly with a severe migraine. Prodrome phase is before headache and consists of different symptoms such as constipation, loss of concentration, insomnia, depression, nausea, dizziness, muscle stiffness, fatigue, food cravings, irritability, sensitivity to light, loss of appetite, heaviness of head, yawning, vomiting, exhausted feeling, and complies with the study conducted by Schoonman et al. explained about these prodrome symptoms such as aura phase embraces photophobia, phonophobia, and giddiness [6]. Hansen et al. conducted a study on migraine, in which they concluded as, during the first 15 min within the onset of aura, 54\% of patients reported headache fulfilling the criteria for migraine [13]. In our study, aura phase was reported before few minutes of attack of migraine symptoms which vary comparatively with that study. In our study, postdrome symptoms are seen in moderate ratios such as fatigue, depression, euphoric mood, loss of concentration, insomnia, dizziness, exhausted feeling, irritability, loss of appetite, indigestion, and nausea because of chronic or inappropriate use of headache medications and comply with the study done by Nicola J. Giffin et al. [14]. As mentioned, there are several comorbidities linked with migraine, because headaches are associated with various disorders that lead to abrupt, severe, and paroxysmal elevations in blood pressure. In our study, we have identified that high blood pressure can cause headaches because it affects the blood-brain barrier. If a person receives treatment to lower their blood pressure, their symptoms will usually improve within an hour. In our study, we found cases of stroke and Meniere's disease as comorbidity to the migraine. Terrin et al. conducted a study on systematic review and found that revision of the literature highlights that migraine with aura is responsible for more than $1 \%$ of all emergency stroke unit evaluations, and our study also reported with some stroke cases [15]. Radtke et al. conducted a study on the, Migraine and Meniere's disease is there a link? Moreover, the study shows a significantly higher lifetime prevalence of migraine in patients with Meniere's disease (56\%) [16]. For migraine, subjects are prescribed equally with NSAIDs and triptans based on patient compliance, and most of them had side effects like weight gain. In our study, we found that patients with migraine are receiving more with prophylactic therapy with calcium channel blockers (35\%), beta-blockers $(33 \%)$, anticonvulsant (24\%), anti-psychotics (1\%), and antidepressants (7\%).

\section{CONCLUSION}

Within the limitations of the present study following conclusions were drawn: Different subtypes of migraine have been observed with migraine without aura being the most and followed by remaining types in every age group and in that females are affected more than males, especially age group between 26 and 45 is seen more with migraine. Subjects are affected with migraine more from rural area than urban area, irrespective of family history, and migraine severity is worsened with some triggering factors in few subjects. In the present study, we finally conclude that subjects with migraine are arriving with different phases, majorly associated features are seen, followed by prodrome phase and postdrome phase and treatment chart are seen with drugs such as beta-blockers, calcium channel blockers, and antidepressants, for treating migraine in our study.

\section{ACKNOWLEDGMENT}

We pay our sincere gratitude to patients, clinicians, and staff for their support and cooperation in completion of the study.

\section{AUTHORS' CONTRIBUTION}

Poojitha Mamindla and Sharanya Mogilicherla have contributed toward literature search, data collection, and manuscript writing; Om Prakash Prasad contributed toward concepts and design of work; and Deepthi Enumula contributed toward concept, design, manuscript editing, and data analysis.

\section{CONFLICTS OF INTEREST}

None of the authors declare conflicts of interest.

\section{AUTHORS FUNDING}

Our study received no specific grant from any funding agency in the public, commercial, nor for-profit sector.

\section{REFERENCES}

1. Pandey B, Bhupendra DR. Migraine. Eur J Biomed Pharm Sci 2017;4:226-30.

2. Headache classification committee of the international headache society (IHS) the international classification of headache disorders, $3^{\text {rd }}$ edition. Cephalalgia 2018;38:1-211.

3. Poojitha M, Sharanya M, Deepthi E, Prasad OP, Sunder AS. A review on migraine Acta Sci Pharm Sci 2019;3:29-42.

4. Teixido M, Carey J. Migraine-more than a headache. Otolaryngol Head Neck Surg 2014;14:1-4.

5. Yadav RK, Kalita J, Misra UK. A study of triggers of migraine in India. Pain Med 2010;11:44-7.

6. Schoonman GG, Evers DJ, Terwindt GM, van Dijk JG, Ferrari MD. The prevalence of premonitory symptoms in migraine: A questionnaire 
study in 461 Patients. Cephalalgia 2006;26:1209-13.

7. Dodick DW. A phase-by-phase review of migraine pathophysiology. Headache 2018;58 Suppl 1:4-16.

8. Nyholt DR, Gillespie NG, Heath AC, Merikangas KR, Duffy DL, Martin NG. Latent class and genetic analysis does not support migraine with aura and migraine without aura as separate entities. Genet Epidemiol 2004;26:231-44.

9. Jensen R, Stovner LJ. Epidemiology and comorbidity of headache. Lancet Neurol 2008;7:354-61.

10. Galletti F, Cupini LM, Corbelli I, Calabresi P, Sarchielli P. Pathophysiological basis of migraine prophylaxis. Prog Neurobiol 2009;89:176-92.

11. Elrington G. Migraine: Diagnosis and management. J Neurol Neurosurg
Psychiatry 2002;72 Suppl 2:ii10-5.

12. Bigal ME, Liberman JN, Lipton RB. Obesity and migraine: A population study. Neurology 2006;66:545-50.

13. Hansen JM, Lipton RB, Dodick DW, Silberstein SD, Saper JR, Aurora SK, et al. Migraine headache is present in the aura phase: A prospective study. Neurology 2012;79:2044-9.

14. Giffin NJ, Lipton RB, Silberstein SD, Olesen J, Goadsby PJ. The migraine postdrome: An electronic diary study. Neurology 2016;87:309-13.

15. Terrin A, Toldo G, Ermani M, Mainardi F, Maggioni F. When migraine mimics stroke: A systematic review. Cephalalgia 2018;38:2068-78.

16. Radtke A, Lempert T, Gresty MA, Brookes GB, Bronstein AM, Neuhauser H. Migraine and Ménière's disease: Is there a link? Neurology 2002;59:1700-4. 